\title{
RAM-PRESSURE STRIPPING ON DWARF GALAXIES
}

\author{
M.A. GÓMEZ-FLECHOSO \\ Observatoire de Genève \\ CH-1290 Sauverny (Switzerland)
}

\begin{abstract}
We use $\mathrm{SPH} / \mathrm{N}$-body simulations to study the ram-pressure stripping of the gas of dwarf galaxies orbiting in a disc galaxy. The effects of the gaseous disc and halo on the satellites are analysed and the results are compared with the observations of the dwarf spheroidals (and dwarf irregulars) in the Local Group. The stripped gas is compared with the High Velocity Clouds. Finally, a model of evolution of the dwarfs is proposed.
\end{abstract}

\section{Introduction}

Some satellite dwarf galaxies in the Local Group (LG) have low or null gas content (Mateo 1998). However, nearly half of them are (dynamically) associated with large reservoirs of atomic gas, not always placed at the dwarf center (e.g., Carignan 1999). A correlation between the dwarf gas content and its distance to the center of the main galaxy (in the LG) exists (Blitz \& Robishaw 2000). The gas reservoirs of the dwarfs and the High Velocity Clouds (HVCs) also seem to be related, as they both have similar properties (Blitz \& Robishaw 2000; Braun \& Burton 1999, 2000).

In this paper, we study the evolution of a dwarf irregulars (dIrrs) into dwarf spheroidal (dSph), when the ram-pressure removes the gas of the dIrrs as they orbit in a disc galaxy. This gas can be identify as a HVC. We also present a semi-analytical method to simulate the stripping process.

\section{The ram-pressure stripping}

The gas of a satellite moving through a gaseous environment (halo) feels a pressure $P \propto \rho_{e} v^{2},\left(\rho_{e}\right.$ is the halo gas density and $v$ the satellite velocity) that can remove the gas from the satellite (Gunn \& Gott 1972). For a satellite cross section $S$ and a satellite gas mass $M_{g}$, the ram-pressure stripping 

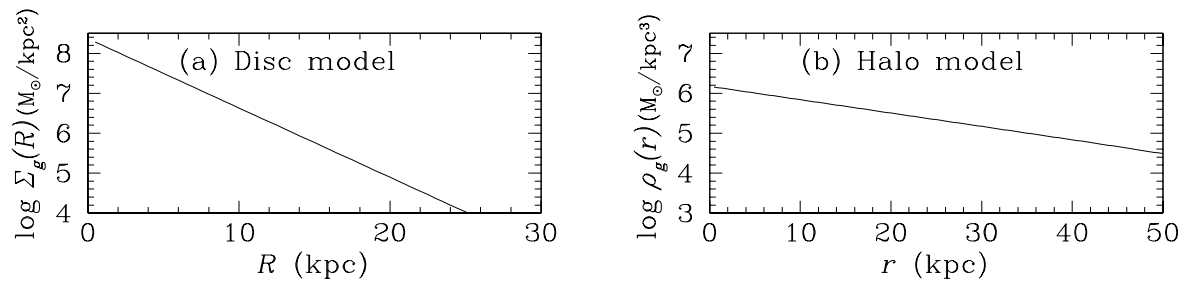

Figure 1. (a) Gas surface density of the disc model, $\Sigma_{g}$, as function of the cylindrical radius, $R$, and (b) gas density of the halo model, $\rho_{g}$, as function of the spherical radius, $r$

of the satellite gas is

$$
\vec{a}_{r p}=\frac{-C_{D} \rho_{e} S v \vec{v}}{2 M_{g}}
$$

where $C_{D} \sim 0.2-1.0$ is a drag parameter.

Taking into account this acceleration and the satellite gravity, the minimum gas density of the halo for the total satellite gas stripping is $\rho_{e}>$ $\alpha G \Sigma_{s} \Sigma_{g} / v^{2}$, where the parameter $\alpha \sim 1$ depends on the geometry of the satellite and $\Sigma_{s}\left(\Sigma_{g}\right)$ is the satellite surface densities of the stars (gas) (Takeda et al 1984; Mori \& Burkert 2000; Blitz \& Robishaw 2000). To obtain a detailed description of the stripping, hydrodynamical simulations of the satellite+halo system are needed. Such simulations are time expensive because they demand a large particle number to describe all the interactions between the satellite and halo and, so far, only the case of a satellite with uniform velocity moving through an uniform halo has been considered (Abadi et al 1999; Mori \& Burkert 2000; Quilis et al 2000).

\section{Model of the ram-pressure stripping}

In order to reduce the calculation time of the simulations, we have described analytically, using a continuous function, the effect of the environment on the (N-body) satellite. We have run simulations using a treesph code (see Barnes \& Hut 1986 and Fux 1997a for details), modified to include the external forces. The gravitational force of the environment affects all the satellite particles and is obtained from the analytical expression of the total environmental density. The ram-pressure acceleration only acts on the gas particles $i$, as

$$
\vec{a}_{r p, i}^{e n v}=\frac{-C_{D} \rho_{e} \pi h_{i}^{2} v_{i} \vec{v}_{i}}{2 m_{i} N_{i}^{1 / 3}}
$$

obtained from eq. (1), where $h_{i}$ is the SPH softening length, $N_{i}$ the number of gas neighbours, $m_{i}$ the mass and $\vec{v}_{i}$ the velocity of the $i$ particle. In the satellite low density regions, $h_{i}$ increases and $N_{i}$ decreases, therefore the 


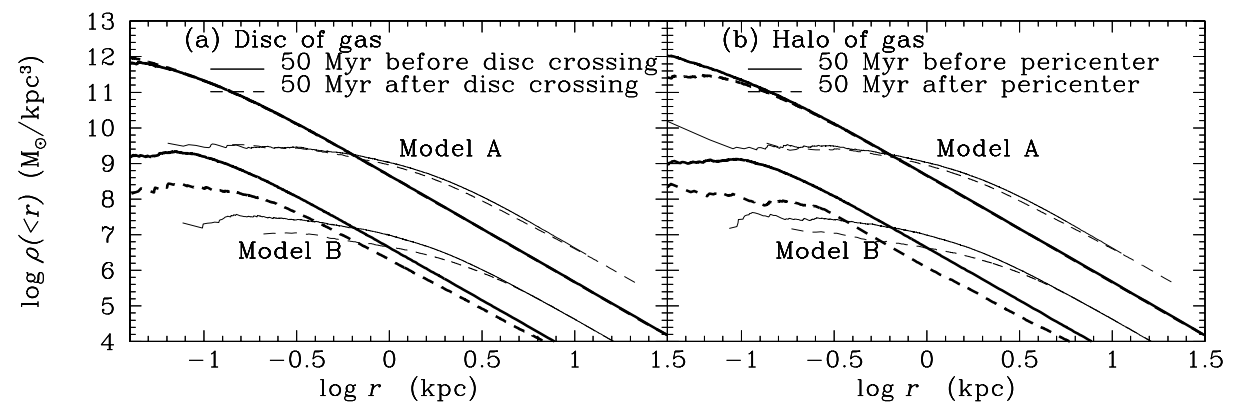

Figure 2. (a) Gas (thick line) and star (thin line) density profiles for the satellite model A (four upper profiles) and model B (four lower profiles), 50 Myrs before (solid line) and after (dashed line) disc crossing, when the gas of the primary galaxy is placed in the disc. (b) Idem that (a) but the gas of the primary galaxy is in the halo.

ram-pressure grows as expected. The contrary occurs in the satellite high density regions. Details of the model are given in Gómez-Flechoso (2000).

\section{Simulations of the satellite accretion}

We have run simulations of an analytical multicomponent (halo+bulge+disc) galaxy which accretes a dwarf satellite. The main galaxy represents a Milky Way like galaxy (see Fux 1997b for details). The gas of the primary galaxy is placed either in the disc or in the halo (densities are plotted in Fig. 1). Our satellites are N-body realizations of a King model (dimensionless central potential $W_{o}=4$ and core radius $r_{o}=1 \mathrm{kpc}$ ), but we include $10 \%$ of its mass as gas in hydrostatic equilibrium. We have considered two satellites: (i) a Large Magellanic Cloud (LMC) like model (model A), massive $\left(M_{\text {sat }}=2 \times 10^{10} \mathrm{M}_{\odot}\right)$ and dense, and (ii) a Sagittarius like model (model B), with low density and $M_{\text {sat }}=2 \times 10^{8} \mathrm{M}_{\odot}$.

In order to give limits to the gas stripping, we have taken a very eccentric orbit perpendicular to the disc (apocenter $R_{a}=70 \mathrm{kpc}$ and pericenter $R_{p}=8 \mathrm{kpc}$ ), as the stripping is higher in high density regions.

\section{Results}

We have study the tidal stripping by a disc galaxy and the ram-pressure stripping by either a disc or a halo of gas on a dwarf satellite model.

(a) Ram-pressure stripping by a disc: The dwarf gas stripping at the disc crossing depends on the dwarf density. Whereas the satellite model A suffers very little stripping, model B loses $\sim 50 \%$ of the gas mass after the first disc crossing and the stripped gas mainly finishes bound to the disc, orbiting with the disc circular velocity. However, the tidal stripping of the 
stars is less significative (density profiles are plotted in Fig. 2a).

(b) Ram-pressure stripping by a halo: The ram-pressure stripping occurs at every point along the orbit, but mainly at the pericenter (where the halo density is higher). As in the previous case, the gas of the model A only suffers a slow evolution. On the contrary, the model B loses almost all the gas $(\sim 70 \%)$ soon after the first pericenter, that forms tails and condensations, whose central surface density and the velocity $\left(\sim 10^{20}\right.$ atoms $/ \mathrm{cm}^{2}$ and $100-300 \mathrm{~km} / \mathrm{s}$, respectively) are similar to those of the HVCs. The tidal stripping is not strong enough to disrupt the satellite, given rise to a dwarf galaxy without gas (density profiles are plotted in Fig. 2b).

\section{Conclusions}

The evolution of a gaseous dwarf galaxy depends on its density:

(a) High density satellites suffer slow evolution and marginal gas losses. That can explain the existence of satellites (as the LMC) with gaseous tails.

(b) Low density satellites lose their gas after a few pericenters, whereas a large percentage of the stellar (and dark) material remains bound to the dwarf, supporting the evolution from dIrrs into dSphs when they orbit in a disc galaxy. It can also explain the apparent correlation between the dwarf gas content and its distance to the primary.

The fate of the stripped gas depends on the distribution of the gas in the primary:

(a) If the gas of the primary is in the halo, the stripped gas of the dwarf forms condensations that can be identified as HVCs.

(b) If the gas is in the disc, the satellite gas is stripped as it crosses the disc and it finishes orbiting in it. It can feed the star formation in the disc.

In a general case, both process (stripping by a halo and by a disc) coexist, increasing the gas loss rate of the dwarf.

\section{References}

Abadi, M.G., Moore, B. \& Bower, R.G. 1999, MNRAS, 308, 947

Barnes, J.E. \& Hut, P. 1986, Nature, 324, 446

Blitz, L. \& Robishaw, T. 2000, astro-ph/0001142

Braun, R. \& Burton, W.B. 1999, A\&A, 341, 347

Braun, R. \& Burton, W.B. 2000, A\&A, 354, 853

Carignan, C. 1999, PASP, 16, 18

Fux, R. 1997a, PhD Thesis

Fux, R. 1997b, A\&A, 327, 983

Gómez-Flechoso, M.A. 2000, in preparation

Gunn, J.E. \& Gott, J.R. 1972, ApJ, 176, 1

Mateo, M. 1998, ARAA, 36, 435

Mori, M. \& Burkert, A. 2000, ApJ, 538, 559

Quilis, V., Moore, B. \& Bower, R. 2000, Science, 288, 1617

Takeda, H., Nulsen, P.E.J. \& Fabian, A.C. 1984, MNRAS, 208, 261 